\title{
The correlation between raised body mass index and assisted reproductive treatment outcomes: a systematic review and meta- analysis of the evidence
}

Prasanna Raj Supramaniam ${ }^{1 *}$, Monica Mittal ${ }^{1}$, Enda McVeigh² and Lee Nai Lim

\begin{abstract}
Background: Public funding for fertility services within the United Kingdom is limited, and therefore, strict guidance exists regarding who can be offered treatment under the National Health Service (NHS). Body mass index (BMI) is a universal criteria adopted by both the public and private sector.

This study addresses an important aspect of the impact of a raised BMI on fertility treatment outcomes. We standardise the analysis of the data by only including studies incorporating the WHO BMI criteria; the current reference point for clinicians and clinical commissioning groups in ascertaining which group of patients should receive treatment. This study is an update of the previous systematic review performed in 2010, with the inclusion of a larger number of cycles from central databases such as the Society for Assisted Reproductive Technology (SART).
\end{abstract}

Methods: An electronic literature search was conducted through the Cochrane, Medline and Embase libraries. Data extraction for each outcome measure was pooled and expressed as an odds ratio with 95\% confidence intervals. Where clinical heterogeneity was evident, the random effects model was used to calculate the risk ratio and a fixed effects model was used for the remaining studies. A $p$ value $<0.05$ was considered statistically significant.

Results: A total of 49 studies have been identified and included in this systematic review. Overweight and obese $\left(\mathrm{BMI} \geq 25 \mathrm{~kg} / \mathrm{m}^{2}\right)$ women have a statistically significant lower live birth rate (OR 0.81,95\% Cl 0.74-0.89, $\left.p<0.00001\right)$ following Assisted Reproductive Technology (ART) when comparisons are drawn to women with a normal BMI. An increase is also demonstrated in the number of miscarriages experienced by women with a BMI $\geq 30 \mathrm{~kg} / \mathrm{m}^{2}(\mathrm{OR} 1.52$, $95 \%$ Cl 1.28-1.81, $p<0.00001)$.

Conclusion: Although this review concludes that a clear impact of BMI on ART outcomes is demonstrated, there remains questions as to the pathophysiology underlying these differences. This review supports the government's stringent criteria regarding BMl categories under which NHS funding is made available for ART, through a clear description of poor reproductive outcomes in women with a BMI $\geq 30 \mathrm{~kg} / \mathrm{m}^{2}$.

Keywords: Body mass index (BMI), Assisted reproductive technology (ART), Overweight, Obese

\footnotetext{
*Correspondence: prasannaraj@doctors.org.uk

'Oxford University Hospitals NHS Foundation Trust, John Radcliffe Hospital,

Headley Way, Headington, Oxford OX3 9DU, UK

Full list of author information is available at the end of the article
} 


\section{Plain English summary}

This study highlights the impact of an overweight or obese female partner on fertility treatment outcomes, in particular focusing on IVF. Women who are overweight or obese have been shown to be less likely to have a life birth outcome from an IVF cycle. They are also more likely to suffer from early miscarriages whilst undergoing fertility treatments.

\section{Main manuscript}

The correlation between raised body mass index and assisted reproductive treatment outcomes: A systematic review and meta-analysis of the evidence.

\section{Background}

Obesity is a major challenge for today's clinicians. In 2016, the World Health Organisation (WHO) [1] stated that a staggering $39 \%$ of adults aged $>18$ years fell into the overweight category, of which $40 \%$ were accounted for by women. Furthermore, $13 \%$ of the adult population were documented to be obese, with women accounting for 15\% (WHO Global Health Observatory Data 2016). A raised body mass index (BMI) has been linked to a number of medical comorbidities, as well as being implicated in having a detrimental impact on the reproductive capacity of women in particular. Women who fall into high BMI categories can present with hypothalamicpituitary ovarian dysfunction and thus, low fecundity rates. In 2011, Rittenberg et al., [2] concluded that women with a BMI $\geq 25.0 \mathrm{~kg} / \mathrm{m}^{2}$ had a lower live birth rate through assisted reproductive treatments (ART) compared with women of a normal BMI. This has been further supported by multiple large studies evaluating the impact of BMI on ART outcomes.

The WHO classification of BMI is widely referred to, and provides standardisation for comparison of research outcomes. A documented BMI of $18.5-24.9 \mathrm{~kg} / \mathrm{m}^{2}$ is considered normal and healthy and the preferred range. A BMI of $25-29.9 \mathrm{~kg} / \mathrm{m}^{2}$ refers to overweight and a BMI $\geq 30 \mathrm{~kg} / \mathrm{m}^{2}$ is considered obese. The latter range is further subdivided into Class $1\left(30.0-34.9 \mathrm{~kg} / \mathrm{m}^{2}\right)$, Class 2 $\left(35.0-39.9 \mathrm{~kg} / \mathrm{m}^{2}\right)$ and Class $3\left(\geq 40.0 \mathrm{~kg} / \mathrm{m}^{2}\right)$.

This paper, considers the current evidence regarding the impact of raised BMI on outcomes following ART treatment. A systematic review and meta-analysis of the available evidence will help provide or refute the current recommendations from the government regarding the allocation of resources for fertility treatment.

\section{Methods}

\section{Search strategy}

Literature searches were conducted through the Cochrane, Embase and Medline libraries (1966-2017). The medical subject headings $(\mathrm{MeSH})$ were generated for two categories: 1. Body mass index (BMI, overweight, obesity); 2. in vitro fertilisation (IVF)/ intracytoplasmic sperm injection (ICSI) (embryo, embryo transfer, ART). All identified papers were reviewed by two authors (PRS and $\mathrm{MM}$ ) independently. All discrepancies, regarding inclusion or exclusion of the data were discussed with a final decision mutually agreed upon.

\section{Study inclusion and exclusion criteria}

All relevant published studies reporting on the effects of BMI on IVF and ICSI pregnancy outcomes were included. Studies that reported donor cycles, conception by natural cycles, intrauterine insemination, waist hip ratio, and non-WHO classification of BMI were excluded. In addition, studies reporting on the effects of paternal body mass index on IVF/ ICSI outcomes were also excluded.

\section{Outcome measures}

The primary outcome measure assessed was live birth rate following an IVF/ ICSI cycle. Secondary outcome measures included: clinical pregnancy rate; and, miscarriage rate. The presence of a gestational sac on an ultrasound scan at least four weeks following on from an embryo transfer was used as confirmation for a clinical pregnancy. The clinical pregnancy rate was calculated per IVF/ ICSI cycle. For the purpose of this review, miscarriage was defined as pregnancy loss $\leq 20$ weeks gestation. The miscarriage rate was calculated per clinical pregnancy.

\section{Statistical analysis}

Data extraction for each outcome measure was pooled and expressed as an odds ratio (OR) with a 95\% confidence interval (CI). Clinical heterogeneity $\left(\mathrm{I}^{2}\right)$ [3] was considered significant when the $\mathrm{I}^{2}$ value was $<50 \%$. Where clinical heterogeneity was evident, the random effects model (DerSimonian and Laird, 1986) was used to calculate the risk ratio, and clinical heterogeneity was explored by comparing the variation in studies, such as, study design, study quality and interventions. Particular care was taken to further evaluate studies with similar first authors to avoid heterogeneity in the study population. For the remaining pooled data, the fixed effect model [4] was used to calculate the risk ratio. Statistical analysis was performed using the RevMan 5.3 software. A $p$ value $<0.05$ was considered statistically significant.

\section{Results}

The search strategy yielded 7458 electronic citations (Fig. 1). Of this, 2830 were removed secondary to duplications. Titles and abstracts were reviewed for the remaining 4628 publications. After screening of the titles and abstracts, 4508 publications were further excluded. 


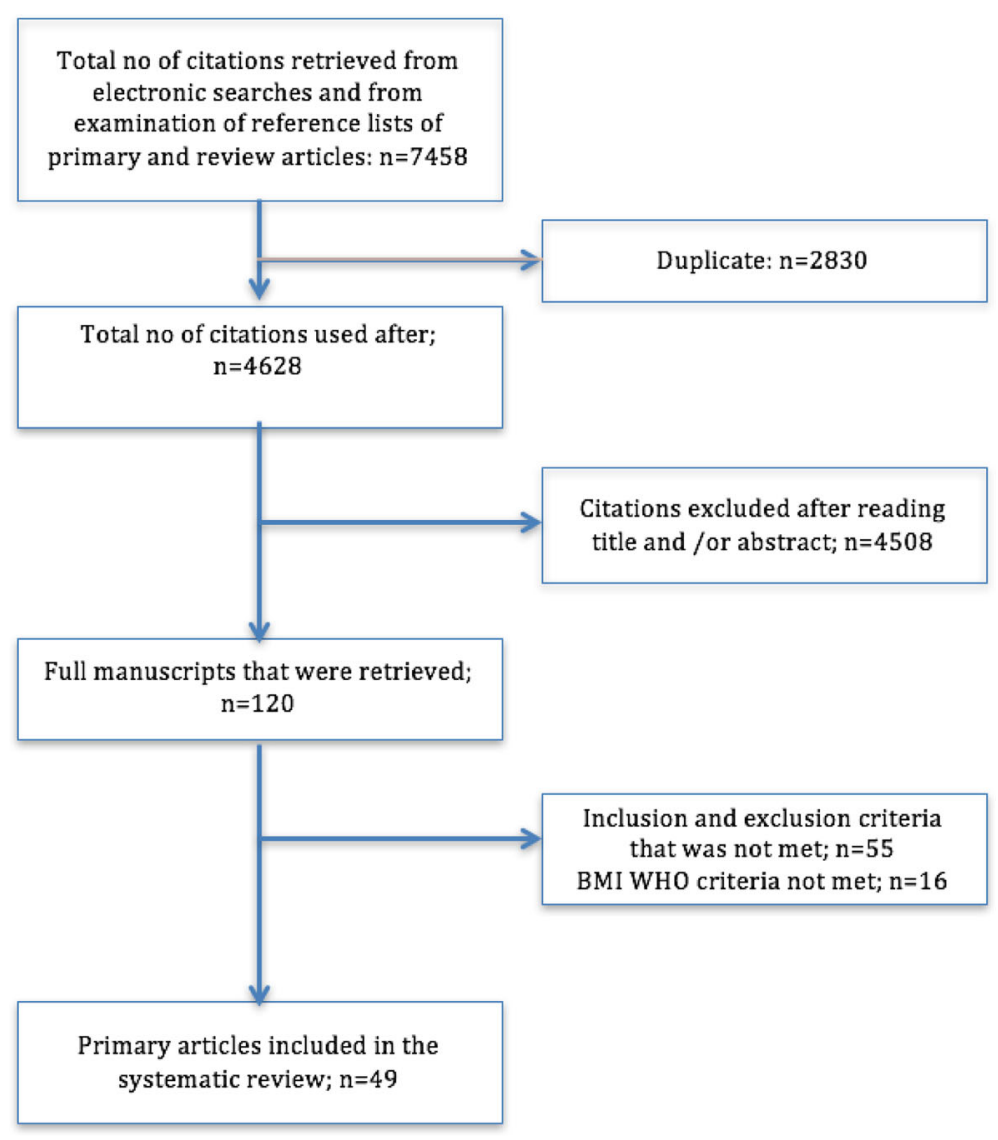

Fig. 1 Flow chart for literature search and study selection

Full manuscripts were obtained for the remaining 120 articles. A further 16 articles were excluded as they did not use the WHO classification for BMI categories. A further 55 articles were excluded as per the inclusion exclusion criteria. The remaining 49 articles met all requirements and were included in this systematic review and meta-analysis (Table 1).

\section{Primary outcome measure} Life birth rate per IVF/ ICSI cycle

In women with a BMI $\geq 25 \mathrm{~kg} / \mathrm{m}^{2}$ versus BMI $<25 \mathrm{~kg} /$ $\mathrm{m}^{2}$, a total of 14 studies were pooled and a statistically significant reduction in the live birth rate (OR 0.81, 95\% CI $0.74-0.89, p<0.00001$; Fig. 2a) was seen. There was significant heterogeneity between the included studies $\left(\mathrm{I}^{2}=65 \%\right)$.

A total of 11 studies compared women with a normal BMI against those who were overweight (BMI $\left.25-29.9 \mathrm{~kg} / \mathrm{m}^{2}\right)$. An analysis of the pooled data showed a statistically significant reduction in the live birth rate in women with a BMI 25-29.9 (OR 0.92, 95\% CI 0.86-0.97, $p=0.005$; Fig. 2b). No significant heterogeneity $\left(\mathrm{I}^{2}=18 \%\right)$ was documented.
Data for women with a normal BMI versus BMI $\geq$ $30 \mathrm{~kg} / \mathrm{m}^{2}$ came from the pooling of 10 studies. The live birth rate for women with a BMI $\geq 30 \mathrm{~kg} / \mathrm{m}^{2}$ was statistically significantly lower than for women with a normal BMI (OR 0.81, 95\% CI 0.79-0.82, $p<0.00001$; Fig. 2c). No significant heterogeneity $\left(\mathrm{I}^{2}=0 \%\right)$ was detected in the data source.

\section{Secondary outcome measures Clinical pregnancy rate}

A total of 37 studies were pooled for BMI $<25 \mathrm{~kg} / \mathrm{m}^{2}$ versus $B M I \geq 25 \mathrm{~kg} / \mathrm{m}^{2}$. A statistically significant reduction in the clinical pregnancy rate was demonstrated for women with a BMI $\geq 25 \mathrm{~kg} / \mathrm{m}^{2}$ (OR $0.82,95 \%$ CI 0.77 $0.88, p<0.00001$; Fig. 3a). However, there was significant heterogeneity $\left(\mathrm{I}^{2}=58 \%, \mathrm{p}<0.00001\right)$ between the studies analysed.

A statistically significant reduction in the clinical pregnancy rate was demonstrated for women with a BMI between 25 and $29.9 \mathrm{~kg} / \mathrm{m}^{2}$ when compared to women with a normal BMI (19 studies pooled, OR 0.89, 95\% CI 0.84-0.94, $p<0.00001$; Fig. 3b). No significant heterogeneity $\left(\mathrm{I}^{2}=31 \%\right)$ was seen between the studies. 
Table 1 Details of included studies

\begin{tabular}{|c|c|c|c|c|c|}
\hline Study & $\begin{array}{l}\text { Methodology } \\
\text { (population size) }\end{array}$ & Intervention & Exclusion Criteria & $\begin{array}{l}\text { BMI Categories }\left(\mathrm{kg} / \mathrm{m}^{2}\right) \\
\text { and numbers }\end{array}$ & $\begin{array}{l}\text { Outcome } \\
\text { Measures }\end{array}$ \\
\hline $\begin{array}{l}\text { Fedorcsak et al. } \\
2000[8] \\
(1996-1998)\end{array}$ & $\begin{array}{l}\text { Retrospective } \\
\text { Cohort study } \\
\text { (383 women) }\end{array}$ & IVF/ICSI & $\begin{array}{l}12 \text { patients excluded } \\
\text { as incomplete data }\end{array}$ & $\begin{array}{l}<25.0 \text { (304 women) } \\
\geq 25.0 \text { (79 women) }\end{array}$ & $\begin{array}{l}\text { Live birth rate } \\
\text { Fertilization rate } \\
\text { No of oocytes } \\
\text { retrieved } \\
\text { Abortion rate }\end{array}$ \\
\hline $\begin{array}{l}\text { Wittemer et al. } \\
2000 \text { [9] } \\
(1997-1998)\end{array}$ & $\begin{array}{l}\text { Retrospective } \\
\text { study } \\
\text { (398 women) }\end{array}$ & IVF/ICSI & None stated & $\begin{array}{l}<20.0 \text { ( } 87 \text { women }) \\
20.0-25.0 \text { ( } 222 \text { women }) \\
\geq 25.0 \text { (89 women) }\end{array}$ & $\begin{array}{l}\text { Pregnancy rate } \\
\text { Delivery rate } \\
\text { Miscarriage rate }\end{array}$ \\
\hline $\begin{array}{l}\text { Wang et al. } \\
2000[10] \\
(1987-1998)\end{array}$ & $\begin{array}{l}\text { Retrospective } \\
\text { study } \\
\text { (3586 women) }\end{array}$ & $\begin{array}{l}\text { IVF/ICSI } \\
\text { and GIFT }\end{array}$ & None stated & $\begin{array}{l}<20.0 \text { (441 women) } \\
\text { 20.0-24.9 (1910 women) } \\
25.0-29.9 \text { (814 women) } \\
\text { 30.0-34.9 (304 women) } \\
\geq 35.0 \text { (117 women) }\end{array}$ & $\begin{array}{l}\text { Probability of achieving } \\
\text { at least one pregnancy }\end{array}$ \\
\hline $\begin{array}{l}\text { Loveland et al. } \\
2001[11] \\
(1997-1999)\end{array}$ & $\begin{array}{l}\text { Retrospective study } \\
\text { (139 women } \\
\text { / } 180 \text { cycles) }\end{array}$ & IVF & $\begin{array}{l}\text { Women }>40 \text { years of age, } \\
\text { blastocyst or frozen embryo } \\
\text { transfer, donor cycles }\end{array}$ & $\begin{array}{l}\leq 25 \\
\text { (70 women / } 87 \text { cycles) } \\
>25 \\
\text { (69 women / } 93 \text { cycles) }\end{array}$ & $\begin{array}{l}\text { Number of oocytes } \\
\text { Clinical pregnancy rate } \\
\text { Spontaneous abortion } \\
\text { Ongoing pregnancy } \\
\text { rate }\end{array}$ \\
\hline $\begin{array}{l}\text { Wang et al. } \\
2001[12] \\
(1987-1999)\end{array}$ & $\begin{array}{l}\text { Cohort study } \\
\text { (1018 women) }\end{array}$ & $\begin{array}{l}\text { IVF/ICSI/ } \\
\text { GIFT }\end{array}$ & $\begin{array}{l}\text { Women whose BMI } \\
\text { or PCOS status was } \\
\text { not assessed }\end{array}$ & $\begin{array}{l}<20.0 \text { (112 women) } \\
\text { 20.0-24.9 (509 women) } \\
25.0-29.9 \text { ( } 231 \text { women) } \\
\text { 30.0-34.9 ( } 116 \text { women) } \\
\geq 35.0 \text { (50 women) }\end{array}$ & Spontaneous abortion \\
\hline $\begin{array}{l}\text { Wang et al. } \\
2002[13] \\
(1987-1999)\end{array}$ & $\begin{array}{l}\text { Retrospective analysis } \\
\text { ( } 2349 \text { women) }\end{array}$ & $\begin{array}{l}\text { IVF/ICSI/ } \\
\text { GIFT }\end{array}$ & $\begin{array}{l}\text { Ectopic pregnancy, } \\
\text { late pregnancy, } \\
\text { women whose BMI was } \\
\text { measured }>\text { /= } 1 \text { year } \\
\text { before pregnancy }\end{array}$ & $\begin{array}{l}<18.5 \text { (70 women) } \\
18.5-24.9 \\
\text { (1508 women) } \\
25-29.9 \text { (503 women) } \\
30-34.9 \text { ( } 198 \text { women) } \\
\geq 35 \text { (70 women) }\end{array}$ & Spontaneous miscarriage \\
\hline $\begin{array}{l}\text { Winter et al. } \\
2002[14] \\
(1994-1999)\end{array}$ & $\begin{array}{l}\text { Cohort } \\
\text { (1123 women / } \\
1196 \text { cycles) }\end{array}$ & $\begin{array}{l}\text { IVF/ICSI/ } \\
\text { GIFT }\end{array}$ & & $\begin{array}{l}<18.5 \text { (26 women) } \\
18.5-25.0 \text { (701 women) } \\
25.1-30.0 \text { ( } 243 \text { women) } \\
\text { 30.1-35.0 (107 women) } \\
>35.0 \text { (46 women) }\end{array}$ & Early pregnancy loss \\
\hline $\begin{array}{l}\text { Doody et al. } \\
2003[15] \\
(2000-2003)\end{array}$ & $\begin{array}{l}\text { Retrospective analysis } \\
\text { (822 retrievals) }\end{array}$ & IVF/ICSI & $\begin{array}{l}\text { Donor cycles, } \\
\text { age }>40 \text { years }\end{array}$ & $\begin{array}{l}<25 \text { (460 women) } \\
25-29.9 \text { (194 women) } \\
\text { 30-34.9 (89 women) } \\
>35 \text { (79 women) }\end{array}$ & $\begin{array}{l}\text { Pregnancy rate } \\
\text { Implantation rate } \\
\text { No of oocytes } \\
\text { No of embryos } \\
\text { transferred } \\
\text { Ongoing } \\
\text { pregnancy rate }\end{array}$ \\
\hline $\begin{array}{l}\text { Fedorscak et al. } \\
2004[16] \\
(1996-2002)\end{array}$ & $\begin{array}{l}\text { Retrospective Study } \\
\text { (2660 women / } \\
5019 \text { cycles) }\end{array}$ & IVF/ICSI & None stated & $\begin{array}{l}<18.5 \\
\text { ( } 76 \text { women/136 cycles) } \\
18.5-24.9 \\
\text { ( } 1839 \text { women/ } \\
3457 \text { cycles) } \\
25.0-29.9 \\
(504 \text { women/ } \\
963 \text { cycles) } \\
\geq 30.0 \\
(241 \text { women/ } \\
463 \text { cycles) }\end{array}$ & $\begin{array}{l}\text { No of oocytes collected } \\
\text { No of embryo transferred } \\
\text { No of embryo transfers } \\
\text { No of biochemical } \\
\text { pregnancies } \\
\text { Early pregnancy loss } \\
\text { Miscarriage } \\
\text { (6-12 weeks), } \\
\text { (> } 12 \text { weeks) } \\
\text { Ectopic pregnancy } \\
\text { Stillbirth } \\
\text { Live birth rate } \\
\text { Dose of FSH } \\
\text { Duration of FSH }\end{array}$ \\
\hline $\begin{array}{l}\text { Ryley et al. } \\
2004 \text { [17] }\end{array}$ & $\begin{array}{l}\text { Retrospective study } \\
\text { (6827 cycles) }\end{array}$ & IVF & Women with $\mathrm{BMI}>40$ & $\begin{array}{l}<20.0 \text { (466 cycles) } \\
20.0-24.9 \text { ( } 3605 \text { cycles) } \\
25.0-29.9 \text { ( } 1632 \text { cycles) } \\
30.0-34.9 \text { ( } 724 \text { cycles) } \\
=35 \text { ( } 400 \text { cycles) }\end{array}$ & $\begin{array}{l}\text { Clinical pregnancy rate } \\
\text { No of oocytes }\end{array}$ \\
\hline $\begin{array}{l}\text { Van Swieten et } \\
\text { al. } 2005 \text { [18] }\end{array}$ & $\begin{array}{l}\text { Observational } \\
\text { (162 women/ } 288 \text { cycle) }\end{array}$ & IVF/ICSI & None stated & $\begin{array}{l}<25 \text { (101 women) } \\
25-30 \text { (32 women) } \\
>30 \text { (29 women) }\end{array}$ & $\begin{array}{l}\text { Fertilisation rate } \\
\text { No oocytes retrieved } \\
\text { Clinical pregnancy rate } \\
\text { Abortion rate }\end{array}$ \\
\hline
\end{tabular}


Table 1 Details of included studies (Continued)

\begin{tabular}{|c|c|c|c|c|c|}
\hline Study & $\begin{array}{l}\text { Methodology } \\
\text { (population size) }\end{array}$ & Intervention & Exclusion Criteria & $\begin{array}{l}\text { BMI Categories }\left(\mathrm{kg} / \mathrm{m}^{2}\right) \\
\text { and numbers }\end{array}$ & $\begin{array}{l}\text { Outcome } \\
\text { Measures }\end{array}$ \\
\hline $\begin{array}{l}\text { Hammadeh et } \\
\text { al. } 2005 \text { [19] }\end{array}$ & $\begin{array}{l}\text { Prospective } \\
\text { (52 women) }\end{array}$ & IVF & None stated & $\begin{array}{l}\leq 25.0 \text { (28 women }) \\
>25.0 \text { ( } 24 \text { women })\end{array}$ & Pregnancy rate \\
\hline $\begin{array}{l}\text { Dechaud et al. } \\
2006[20]\end{array}$ & $\begin{array}{l}\text { Prospective study } \\
\text { (573 women/ } 789 \text { cycles) }\end{array}$ & IVF/ICSI & $\begin{array}{l}\text { Women with a history of } \\
\text { uterine surgery, hydrosalpinges } \\
\text { evidenced by ultrasonography, } \\
\text { three or more failed attempts } \\
\text { at IVF, frozen-thawed cycles, } \\
\text { women undergoing } \\
\text { pre-implantation diagnosis and } \\
\text { those using a protocol other } \\
\text { than the long protocol }\end{array}$ & $\begin{array}{l}<20 \\
\text { (186 women/ } \\
264 \text { cycles) } \\
20-25 \text { ( } 283 \text { women/ } \\
394 \text { cycles) } \\
25-30 \text { ( } 68 \text { women/ } \\
83 \text { cycles) } \\
\geq 30 \text { ( } 36 \text { women/ } \\
48 \text { cycles) }\end{array}$ & $\begin{array}{l}\text { Duration of ovarian } \\
\text { stimulation } \\
\text { Dose of FSH } \\
\text { Implantation rate } \\
\text { No of oocytes } \\
\text { Fertilization rate } \\
\text { Clinical pregnancy rate } \\
\text { Miscarriage rate }\end{array}$ \\
\hline $\begin{array}{l}\text { Dokras et al. } \\
2006[21] \\
(1995-2005)\end{array}$ & $\begin{array}{l}\text { Retrospective } \\
\text { Study } \\
\text { (1293 women) }\end{array}$ & $\begin{array}{l}\text { IVF/IVF } \\
\text { with ICSI }\end{array}$ & $\begin{array}{l}\text { Women }>38 \text { years of age, } \\
\text { day } 2 \text { transfer cycles, } \\
\text { cryopreserved embryo transfers, } \\
\text { donor oocyte cycle, gamete } \\
\text { intrafallopian transfer and zygote } \\
\text { intrafallopian transfer cycles }\end{array}$ & $\begin{array}{l}<25 \text { (683 women) } \\
25-29.9 \text { (295 women) } \\
30.0-39.9 \text { ( } 236 \text { women) } \\
\geq 40 \text { (79 women) }\end{array}$ & $\begin{array}{l}\text { No of follicles aspirated } \\
\text { Fertilization rate } \\
\text { No of embryo(s) } \\
\text { transferred } \\
\text { Clinical pregnancy rate } \\
\text { Miscarriage rate } \\
\text { Delivery rate } \\
\text { Days of stimulation }\end{array}$ \\
\hline $\begin{array}{l}\text { Mitwally et al. } \\
2006 \text { [22] }\end{array}$ & $\begin{array}{l}\text { Cohort } \\
\text { (183 cycles) }\end{array}$ & IVF & None stated & $\begin{array}{l}<25.0 \text { ( } 102 \text { cycles }) \\
\geq 25.0 \text { (81 cycles) }\end{array}$ & Clinical pregnancy rate \\
\hline $\begin{array}{l}\text { Metwally } 2007 \\
{[23]} \\
(2001-2006)\end{array}$ & $\begin{array}{l}\text { Retrospective analysis } \\
\text { ( } 426 \text { women) }\end{array}$ & IVF/ICSI & $\begin{array}{l}\text { Cycles on women whose } \\
\text { BMI was unrecorded }\end{array}$ & $\begin{array}{l}19-24.9 \text { (241 women) } \\
25-29.9 \text { ( } 113 \text { women) } \\
\geq 30 \text { (72 women) }\end{array}$ & $\begin{array}{l}\text { Fertilization rate } \\
\text { Clinical pregnancy rate } \\
\text { Dose of FSH } \\
\text { Duration of FSH } \\
\text { No of oocytes collected }\end{array}$ \\
\hline $\begin{array}{l}\text { Esinler et al. } \\
2008 \text { [24] }\end{array}$ & $\begin{array}{l}\text { Retrospective } \\
\text { Study } \\
\text { (775 women/ } \\
1113 \text { cycles) }\end{array}$ & $|C S|$ & $\begin{array}{l}\text { Freeze-thaw cycles, female } \\
\text { age }>40 \text {, presence of PCOS, } \\
\text { history of irregular menstrual } \\
\text { cycle and suspected poor } \\
\text { ovarian response }\end{array}$ & $\begin{array}{l}18.5-24.9 \\
(451 \text { women/ } \\
627 \text { cycles }) \\
25.0-29.9 \\
(222 \text { women/ } \\
339 \text { cycles }) \\
\geq 30.0 \\
\text { (102 women/ } \\
147 \text { cycles) }\end{array}$ & $\begin{array}{l}\text { Clinical pregnancy rate } \\
\text { Fertilization rate } \\
\text { No of miscarriages } \\
\text { No of oocytes } \\
\text { Dose of FSH } \\
\text { Duration of FSH }\end{array}$ \\
\hline $\begin{array}{l}\text { Martinuzzi et al. } \\
2008[25] \\
(2004-2006)\end{array}$ & $\begin{array}{l}\text { Retrospective } \\
\text { study } \\
\text { ( } 417 \text { women) }\end{array}$ & IVF & $\begin{array}{l}\text { Women }>36 \text { years of age, } \\
\text { cycle day-3 }\end{array}$ & $\begin{array}{l}<18.5 \text { (21 women) } \\
18.5-24.9 \text { ( } 267 \text { women) } \\
25.0-29.9 \text { ( } 77 \text { women) } \\
\geq 30 \text { (52 women) }\end{array}$ & $\begin{array}{l}\text { No of oocytes } \\
\text { Fertilization rate } \\
\text { Implantation rate } \\
\text { Clinical pregnancy rate } \\
\text { Ongoing pregnancy rate }\end{array}$ \\
\hline $\begin{array}{l}\text { Moini et al. } \\
2008[26] \\
(2002-2003)\end{array}$ & $\begin{array}{l}\text { Cross-sectional } \\
\text { study } \\
\text { ( } 287 \text { women) }\end{array}$ & IVF/ICSI & $\begin{array}{l}\text { Women who did not have } \\
\text { polycystic ovary syndrome, } \\
\text { age }>40 \text { years, } \mathrm{BMI}<20 \text {, } \\
\text { women with hypo/ } \\
\text { hyperthyroidism, } \\
\text { hyperprolactinemia and } \\
\text { diabetes type } 1\end{array}$ & $\begin{array}{l}\text { 20-25 (133 women) } \\
25.1-30 \text { ( } 117 \text { women) } \\
>30 \text { (37 women) }\end{array}$ & $\begin{array}{l}\text { No of oocytes } \\
\text { No of transferred embryos } \\
\text { Clinical pregnancy rate } \\
\text { Miscarriage rate }\end{array}$ \\
\hline $\begin{array}{l}\text { Sneed et al. } \\
2008[27] \\
(2005-2006)\end{array}$ & $\begin{array}{l}\text { Retrospective analysis } \\
\text { (1273 women) }\end{array}$ & IVF & $\begin{array}{l}\text { Frozen cycles, donor } \\
\text { oocyte or gestational } \\
\text { surrogacy cycles, age } \\
>44 \text { years }\end{array}$ & $\begin{array}{l}<18.5 \text { ( } 28 \text { women) } \\
>18.5-24.9 \text { (613 women) } \\
>25-29.9 \\
\text { (325 women) } \\
>30 \\
\text { (307 women) }\end{array}$ & $\begin{array}{l}\text { No of oocytes } \\
\text { No of embryo transfers } \\
\text { Fertilization rate } \\
\text { Implantation rate } \\
\text { Spontaneous abortion } \\
\text { Clinical pregnancies } \\
\text { Live birth rate }\end{array}$ \\
\hline $\begin{array}{l}\text { Ozgun et al. } \\
2009[28] \\
(2006-2007)\end{array}$ & $\begin{array}{l}\text { Prospective study } \\
\text { (604 women) }\end{array}$ & $|\mathrm{CS}|$ & $\begin{array}{l}\text { Women > } 42 \text { years old, medical } \\
\text { co-morbidities such as diabetes } \\
\text { mellitus, hyper or hypothyroidism, } \\
\text { basal FSH > } 15 \mathrm{IU} / \mathrm{L} \text {, thawed } \\
\text { embryo transfer cycles, history } \\
\text { of prior ovarian surgery, poor } \\
\text { responders, couples with more } \\
\text { than one etiology for their } \\
\text { infertility }\end{array}$ & $\begin{array}{l}<18.5 \\
\text { (10 women) } \\
18.5-24.9 \\
\text { ( } 232 \text { women) } \\
25-29.9 \\
\text { ( } 229 \text { women) } \\
30-35.9 \\
\text { (111 women) } \\
\geq 36 \\
\text { ( } 22 \text { women) }\end{array}$ & $\begin{array}{l}\text { No of Pregnancy } \\
\text { Total FSH dosage }\end{array}$ \\
\hline
\end{tabular}


Table 1 Details of included studies (Continued)

\begin{tabular}{|c|c|c|c|c|c|}
\hline Study & $\begin{array}{l}\text { Methodology } \\
\text { (population size) }\end{array}$ & Intervention & Exclusion Criteria & $\begin{array}{l}\text { BMl Categories }\left(\mathrm{kg} / \mathrm{m}^{2}\right) \\
\text { and numbers }\end{array}$ & $\begin{array}{l}\text { Outcome } \\
\text { Measures }\end{array}$ \\
\hline $\begin{array}{l}\text { Sathya et al. } \\
2010 \text { [29] }\end{array}$ & $\begin{array}{l}\text { Retrospective study } \\
\text { (308 women) }\end{array}$ & IVF & $\begin{array}{l}\text { Women }>40 \text { years of age, } \\
\mathrm{FSH}>10 \mathrm{mlU} / \mathrm{ml}\end{array}$ & $\begin{array}{l}<25 \\
(88 \text { women) } \\
25-30 \\
\text { (147 women) } \\
>30 \\
\text { (73 women) }\end{array}$ & $\begin{array}{l}\text { No of embryos transferred } \\
\text { Clinical pregnancy rate } \\
\text { Missed abortion rate } \\
\text { Multiple pregnancy rate } \\
\text { Ectopic pregnancy rate } \\
\text { Implantation rate } \\
\text { Gonadotrophin dosage }\end{array}$ \\
\hline $\begin{array}{l}\text { Zhang et al. } \\
2010 \text { [30] (2002- } \\
\text { 2008) }\end{array}$ & $\begin{array}{l}\text { Retrospective study } \\
\text { (2628 women) }\end{array}$ & IVF/ICSI & $\begin{array}{l}\text { Patients with severe } \\
\text { endometriosis (III and IV stage) } \\
\text { diagnosed by laparoscopy, } \\
\text { more than two failed previous } \\
\text { attempts, preimplantation } \\
\text { diagnosis cycles, frozen } \\
\text { thawed cycles, protocols other } \\
\text { than the long protocol }\end{array}$ & $\begin{array}{l}18.5-24.9 \\
(2222 \text { women) } \\
25.0-29.9 \\
\text { (379 women) } \\
\geq 30.0 \\
\text { ( } 27 \text { women) }\end{array}$ & $\begin{array}{l}\text { No of oocytes } \\
\text { Fertilization rate } \\
\text { Pregnancy rate } \\
\text { Early pregnancy loss rate } \\
\text { Ectopic pregnancy } \\
\text { Miscarriage rate } \\
\text { Live birth rate } \\
\text { Days of FSH stimulation } \\
\text { Dosage of FSH } \\
\text { stimulation } \\
\text { Ongoing pregnancy rate }\end{array}$ \\
\hline $\begin{array}{l}\text { Bellver et al. } \\
2010[31] \\
(2001-2007)\end{array}$ & $\begin{array}{l}\text { Retrospective study } \\
\text { (6500 cycles) }\end{array}$ & IVF/ICSI & None stated & $\begin{array}{l}<20 \\
\text { (669 women / } \\
1070 \text { cycles) } \\
20-24.9 \\
\text { (2620 women/ } \\
3930 \text { cycles) } \\
25-29.9 \\
\text { (676 women/ } \\
1081 \text { cycles) } \\
\geq 30 \\
\text { (262 cycles/ } \\
419 \text { cycles) }\end{array}$ & $\begin{array}{l}\text { Total dose of } \\
\text { gonadotrophin } \\
\text { No of oocytes } \\
\text { Fertilization rate } \\
\text { No of embryos transferred } \\
\text { Implantation rate } \\
\text { Pregnancy rate } \\
\text { Clinical pregnancy rate } \\
\text { Clinical and global } \\
\text { miscarriage rate } \\
\text { Live birth rate }\end{array}$ \\
\hline $\begin{array}{l}\text { Vilarino et al. } \\
2010[32] \\
(2008)\end{array}$ & $\begin{array}{l}\text { Retrospective } \\
\text { (208 cycles/ } 191 \text { women) }\end{array}$ & IVF/ICSI & $\begin{array}{l}\text { Frozen and donor } \\
\text { oocyte-derived cycles }\end{array}$ & $\begin{array}{l}<25 \text { (137 cycles }) \\
\geq 25 \text { (71 cycles) }\end{array}$ & $\begin{array}{l}\text { Fertilisation rate } \\
\text { No of transferred embryos } \\
\text { Pregnancy rate } \\
\text { Early pregnancy loss } \\
\text { Clinical miscarriage rate } \\
\text { Ectopic pregnancy } \\
\text { Live birth rate } \\
\text { Dosage of FSH }\end{array}$ \\
\hline $\begin{array}{l}\text { Farhi et al. } 2010 \\
\text { [33] } \\
(2006-2007)\end{array}$ & $\begin{array}{l}\text { Retrospective study } \\
\text { (233 women/ } 233 \text { cycles) }\end{array}$ & IVF & $\begin{array}{l}\text { Women } \geq 38 \text { years of age, } \\
\text { other than } 2 \text { high-quality embryos, } \\
\geq 3 \text { previous IVF attempts, women } \\
\text { with hydrosalpinx, fibroid uterus, } \\
\text { congenital uterine anomaly } \\
\text { and chronic illness }\end{array}$ & $\begin{array}{l}\leq 25.0 \text { (160 women) } \\
>25.0 \text { (73 women) }\end{array}$ & $\begin{array}{l}\text { Live birth rate } \\
\text { Pregnancy rate } \\
\text { No of oocytes } \\
\text { Fertilization rate }\end{array}$ \\
\hline $\begin{array}{l}\text { Davies et al. } \\
2010 \text { [34] (2008- } \\
2009)\end{array}$ & 232 cycles & IVF & $\begin{array}{l}\text { Donor egg, gestational carrier } \\
\text { and pre-implantation genetic } \\
\text { diagnosis cycles }\end{array}$ & $\begin{array}{l}<25.0 \text { ( } 176 \text { cycles }) \\
>25.0 \text { (56 cycles) }\end{array}$ & Fetal heartbeat rates \\
\hline $\begin{array}{l}\text { Funabiki et al. } \\
2011 \text { [35] (2006- } \\
2010)\end{array}$ & $\begin{array}{l}\text { Retrospective study } \\
\text { (859 women) }\end{array}$ & IVF & None stated & $\begin{array}{l}<18.5 \text { ( } 152 \text { women }) \\
18.5-25.0 \text { ( } 648 \text { women }) \\
\geq 25.0 \text { (59 women) }\end{array}$ & $\begin{array}{l}\text { Pregnancy rate } \\
\text { Ongoing pregnancy rate } \\
\text { Miscarriage rate } \\
\text { No of oocytes }\end{array}$ \\
\hline $\begin{array}{l}\text { Hill et al. } 2011 \\
\text { [36] }\end{array}$ & $\begin{array}{l}\text { Prospective study } \\
\text { (117 women) }\end{array}$ & IVF & $\begin{array}{l}\text { Women }>42 \text { years of age, } \\
\text { patients with elevated FSH } \\
\text { levels }(\geq 12 \mathrm{mIU} / \mathrm{mL})\end{array}$ & $\begin{array}{l}<25.0 \text { (58 women) } \\
\geq 25.0 \text { (59 women) } \\
<30.0 \text { (96 women) } \\
\geq 30.0 \text { ( } 21 \text { women) }\end{array}$ & $\begin{array}{l}\text { Live birth rate } \\
\text { Pregnancy rate } \\
\text { Implantation rate } \\
\text { No of oocytes } \\
\text { No of embryo transferred } \\
\text { Days of stimulation }\end{array}$ \\
\hline
\end{tabular}


Table 1 Details of included studies (Continued)

\begin{tabular}{|c|c|c|c|}
\hline Study & $\begin{array}{l}\text { Methodology } \\
\text { (population size) }\end{array}$ & Intervention & Exclusion Criteria \\
\hline $\begin{array}{l}\text { Pinborg et al. } \\
2011[37] \\
(2005-2006)\end{array}$ & $\begin{array}{l}\text { Cohort study } \\
\text { (487 women/ } 1417 \text { cycles) }\end{array}$ & $\begin{array}{l}\text { IVF/ ICSI/ } \\
\text { FET }\end{array}$ & $\begin{array}{l}\text { Patients undergoing intrauterine } \\
\text { insemination cycles, patients with } \\
\text { an existing child from fertility } \\
\text { treatment, couples who had } \\
\text { adopted a child in the } 12 \text { th month } \\
\text { follow-up period and couples who } \\
\text { had no treatment during the first } \\
12 \text { months of follow up }\end{array}$ \\
\hline
\end{tabular}

$\begin{array}{ll}\begin{array}{l}\text { BMI Categories }\left(\mathrm{kg} / \mathrm{m}^{2}\right) \\ \text { and numbers }\end{array} & \begin{array}{l}\text { Outcome } \\ \text { Measures }\end{array} \\ <18.5 \text { (20 women) } & \text { Fertilization rate } \\ \text { 18.5-24.9 (305 women) } & \text { No of oocytes } \\ 25.0-29.9 \text { (103 women) } & \text { Biochemical } \\ \geq 30.0 \text { (59 women) } & \text { pregnancy rate } \\ & \text { Ectopic pregnancy rate } \\ & \text { Ongoing pregnancy rate } \\ & \text { Miscarriage rate } \\ & \text { Live birth rate } \\ & \text { Dose of gonadotrophin } \\ & \text { stimulation } \\ & \text { No of oocytes } \\ <18.5 \text { (18 women) } & \text { Clinical pregnancy rate } \\ 18.5-24.9 \text { (475 women) } & \text { Implantation rate } \\ 25-29.9(241 \text { women }) & \text { Ongoing pregnancy rate } \\ >30(221 \text { women) } & \text { Total FSH dosage } \\ & \text { No of embryo transferred }\end{array}$

Rittenberg et al. Cohort Study (413 women) IVF/ICSI 2011 [39]

(2006-2010)

Retrospective study IVF/ICSI None stated

2011 [38]

(995 patients)

(2010-2011)

Singh et al.

2011 [40]

(2008-2010)

Retrospective Study

IVF/ICSI

(328 women/ 342 cycles)

2011 [41]

Historical cohort study

(152,500 cycles)

IVF

(2007-2008)

Chavarro et al.
2012 [42]

Prospective study

(170 women/ 233 cycles)

(2004-2011)

Galal et al. 2012 Prospective cohort

[43]

(220 women)

Werner et al. Retrospective study

2012 [44] (2008- (355 women)

2012)

Zander-Fox et Retrospective study

al. 2012 [45] (2089 cycles)

(2006-2007)

Ozgun et al.

2012 [46]

(2005-2010)

$|C S|$

IVF

IVF/ICSI

ICSI (935 women)
Women $>40$ years, $\mathrm{BMl}<18.5$,

$\mathrm{BMI}>35$, pre-implantation genetic

diagnosis, donor oocyte or

embryos frozen for fertility

preservation prior to cancer

therapy cycles, mullerian duct

anomalies, monozygotic

twin gestations

18.5-24.9 (192 women)

$\geq 25$ (133 women)

Oocyte fertilisation rate

No of oocytes

Clinical pregnancy rate

Live birth rate

Miscarriage rate

Duration of stimulation previous failed attempts, frozen thawed cycles

Women whose height and weight were not recorded, gestational carrier cycles, research or embryo banking with no outcome reported

IVF/ICSI Women $<18$ and

$>45$ years of age

None stated

None stated

Women $>38$ years of age, natural and donor cycles

Retrospective cohort
No exclusion criteria
$<18.5$ (26 women) 18.5-24.9 (141 women) 25-29.9 (131 women) > 30 (18 women)

Fertilisation rate

Pregnancy rate

Total dose of FSH

Total days of stimulation

No of oocytes retrieved

Fertilization rate

Clinical pregnancy rate

$<18.5$ (4254 cycles)

Pregnancy rate

$18.5-24.9$ (86,860 cycles)
$25-29.9$ (35,452 cycles)

30.0-34.9 (15,406 cycles)

35.0-39.9 (6920 cycles)

40.0-44.9 (2513 cycles)

45.0-49.9 (805 cycles)

$<20$ (22 women)

22.5-24.9 (42 women)

25-29.9 (35 women)

$\geq 30$ (24 women)

$<25.0$ (110 women)

$>25.0$ (110 women)

$<18.5$ (13 women)

18.5-24.9 (209 women)

25.0-29.9 (88 women)

$>30.0$ (45 women)

18.5-24.9 (1065 cycles)

25.0-29.9 (486 cycles)

30.0-34.9 (244 cycles)

35.0-39.9 (144 cycles)

$\geq 40.0$ (118 cycles)

< 18.5 (18 women)

18.5-24.9 (398 women)

25-29.9 (355 women)

$\geq 30$ (164 women)
Clinical pregnancy rate Total gonadotrophin dose Fertilization rate

Clinical pregnancy rate Live birth rate

No of oocytes

Fertilization rate

Clinical pregnancy rate

No of embryos transferred

Pregnancy rate

Clinical implantation rate

Sustained implantation

rate

No of oocytes

Fertilisation rate

Live delivery

Clinical pregnancy

No of oocytes

Clinical pregnancy rateNo

of oocytes

Miscarriage rate

Total gonadotrophin dose
20-22.4 (47 women) 
Table 1 Details of included studies (Continued)

\begin{tabular}{|c|c|c|c|}
\hline Study & $\begin{array}{l}\text { Methodology } \\
\text { (population size) }\end{array}$ & Intervention & Exclusion Criteria \\
\hline $\begin{array}{l}\text { Ramezanzadeh } \\
\text { et al. } 2012 \text { [47] } \\
(2010-2011)\end{array}$ & $\begin{array}{l}\text { Prospective study } \\
\text { (236 women) }\end{array}$ & IVF & $\begin{array}{l}\text { Male factor infertility according } \\
\text { to the WHO criteria, presence } \\
\text { of systemic disease, age }<18 \text { years } \\
\text { or }>40 \text { years and donor oocytes }\end{array}$ \\
\hline $\begin{array}{l}\text { Moragianni et al. } \\
2012 \text { [48] } \\
(2007-2008)\end{array}$ & $\begin{array}{l}\text { Retrospective } \\
\text { cohort study } \\
\text { (4609 women) }\end{array}$ & $\begin{array}{l}\text { IVF/ } \\
\text { IVF-ICSI }\end{array}$ & $\begin{array}{l}\text { Women }<20 \text { years and }>47 \text { years } \\
\text { of age, donor oocytes, gestational } \\
\text { surrogacy, cryopreserved embryos } \\
\text { or those that lacked BMl } \\
\text { documentation }\end{array}$ \\
\hline
\end{tabular}

\begin{abstract}
Bailey et al. 2014 Retrospective Cohort [49] Study (79 women / (2001-2010) 101 cycles)
\end{abstract}

IVF/ICSI

Women $<40$ years of age, height and weight measurements

$>3$ months from the start of cycle, in-vitro maturation,

$\mathrm{FSH}>10 \mathrm{mlU} / \mathrm{mL}$, uncontrolled thyroid disease, history of chemotherapy or radiation exposure, recurrent pregnancy loss, uterine factor, balanced translocation in either partner, surgically documented endometriosis or pelvic adhesions, history of pelvic inflammatory disease, adenomyosis and submucosal myoma

Schliep et al. Prospective Cohort Study IVF/ICSI 2014 [50] (2005-2010)

Cai et al. 2017

[51]

(2013-2014)

Retrospective Cohort Study IVF/ICSI

(4401 women /

4798 fresh transfer cycles

$\begin{array}{lll}\begin{array}{l}\text { Ozekinci et al. } \\ 2015[52]\end{array} & \begin{array}{l}\text { Retrospective Cohort Study } \\ \text { (298 women) }\end{array} & \text { IVF-ICS } \\ \begin{array}{l}2008-2013) \\ \text { Caillon et al. }\end{array} & \text { Retrospective study } & \text { IVF-ICS } \\ 2015 \text { [53] } & \text { (582 women) } & \\ (2006-2009) & & \\ \text { Provost et al. } & \text { Retrospective Cohort Study } & \text { IVF } \\ 2016 \text { [54] } & \text { (239,127 cycles) } & \end{array}$

2016 [54]

(239,127 cycles)
Men with non-obstructive azoospermia

Mild stimulation cycles, natural cycles and luteal-phase stimulation cycle, patients with diabetes, glucose intolerance and thyroid abnormality

Underweight women, women $>38$ years of age, transfer of $>2$ embryos, frozen cycles

Underweight women

Women with a height $<48$ in. and weight $<70$ pounds

$<18.5$ ( 92 women)
18.5-24.99 ( 2605
women)
25.0-29.99 ( 1027
women)
30.00-34.99 ( 477
women)
35.00-39.99 ( 275
women)
$>40.0$ ( 133 women)

18.7-24.9 (51 cycles) 25.0-29.9 (19 cycles) $\geq 30.0$ (31 cycles)

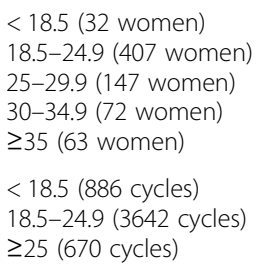

18.5-24.9 (164 cycles) 25-29.9 (70 cycles) $\geq 30$ (64 cycles)

18.5-24.9 (409 women) $\geq 25$ (149 women)

$<18.5$ (7149 cycles)

$18.5-24.9$

$(134,588$ cycles $)$

25-29.9 (54,822 cycles)

30-34.9 (24,922 cycles)

35-39.9 (11,747 cycles)

40-44.9 (4084 cycles)

45-49.9 (1292 cycles)

$>50$ (463 cycles)

Congenital uterine anomalies, endometrial polyps, intrauterine synechiae, adenomyosis, intra-cavity fibroids, hydrosalpinges, donor cycles, poor quality embryos, cleavage stage embryos, and women $>40$ years
$<20$ (51 women) 20-24.9 (294 women) 25-29.9 (64 women) 30-39.9 (58 women) $\geq 40$ (54 women)
Outcome

Measures

No of oocytes Fertilization rate No of embryo transferred Biochemical pregnancies Clinical pregnancy rate Implantation rate

No of oocytes retrieved Duration of stimulation Total dosage of gonadotrophin No of embryo transferred Implantation

Clinical pregnancy Biochemical pregnancy

Global miscarriage Ectopic pregnancy Live birth Multiple birth

Chemical pregnancy Miscarriage

Clinical Pregnancy Live Birth rate

Duration of stimulation of gonadotrophin Dosage of gonadotrophin No of oocytes retrieved

Fertilization rate Pregnancy rate Live birth rate

Fertilization rate Live birth rate

Miscarriage rate Dosage of gonadotrophin

Dosage of gonadotrophin Duration of stimulation

Dosage of gonadotrophin Implantation rate Miscarriage rate Live birth rate

Implantation rate Clinical pregnancy rate Miscarriage rate Live birth rate

Miscarriage rate Clinical pregnancy rate Live birth rate Dosage of gonadotrophin 
Table 1 Details of included studies (Continued)

\begin{tabular}{|c|c|c|c|c|c|}
\hline Study & $\begin{array}{l}\text { Methodology } \\
\text { (population size) }\end{array}$ & Intervention & Exclusion Criteria & $\begin{array}{l}\text { BMl Categories }\left(\mathrm{kg} / \mathrm{m}^{2}\right) \\
\text { and numbers }\end{array}$ & $\begin{array}{l}\text { Outcome } \\
\text { Measures }\end{array}$ \\
\hline $\begin{array}{l}\text { Christensen et } \\
\text { al. } 2016 \text { [56] } \\
(1999-2009)\end{array}$ & $\begin{array}{l}\text { Retrospective Cohort Study } \\
\text { (5342 cycles) }\end{array}$ & IVF/ICSI & $\begin{array}{l}\text { Missing information on BMI or } \\
\text { treatment type, premature ovulation } \\
\text { before oocyte retrieval, intrauterine } \\
\text { insemination cycles }\end{array}$ & $\begin{array}{l}<18.5 \text { ( } 158 \text { cycles }) \\
18.5-24.9 \text { (3539 cycles) } \\
25-29.9 \text { ( } 1171 \text { cycles }) \\
\geq 30 \text { ( } 474 \text { cycles })\end{array}$ & $\begin{array}{l}\text { Dosage of gonadotrophin } \\
\text { Clinical pregnancy rate }\end{array}$ \\
\hline
\end{tabular}

BMI Body Mass Index, IVF in vitro fertilization, ICSI intracytoplasmic sperm injection, OHSS ovarian hyperstimulation syndrome, GIFT gamete intra-Fallopian transfer, HCG human chorionic gonadotrophin, FSH follicle stimulation hormone

Pooled analysis from 18 studies demonstrated a statistically significant reduction in the clinical pregnancy rate for women with a BMI $\geq 30 \mathrm{~kg} / \mathrm{m}^{2}$ when compared to women with a normal BMI (OR 0.80, 95\% CI 0.74-0.87, $p<0.00001$; Fig. 3c). There was no significant heterogeneity $\left(\mathrm{I}^{2}=32 \%\right)$ present between the studies.

\section{Miscarriage rate}

An increased risk of miscarriage is demonstrated in women with a BMI $\geq 25 \mathrm{~kg} / \mathrm{m}^{2}$ when compared to women with a $\mathrm{BMI}<25 \mathrm{~kg} / \mathrm{m}^{2}$ (26 studies pooled, OR 1.30 , 95\% CI 1.15$1.48, p<0.0001$; Fig. 4a). However, significant heterogeneity $\left(\mathrm{I}^{2}=53 \%, p=0.0001\right)$ was seen between the studies.

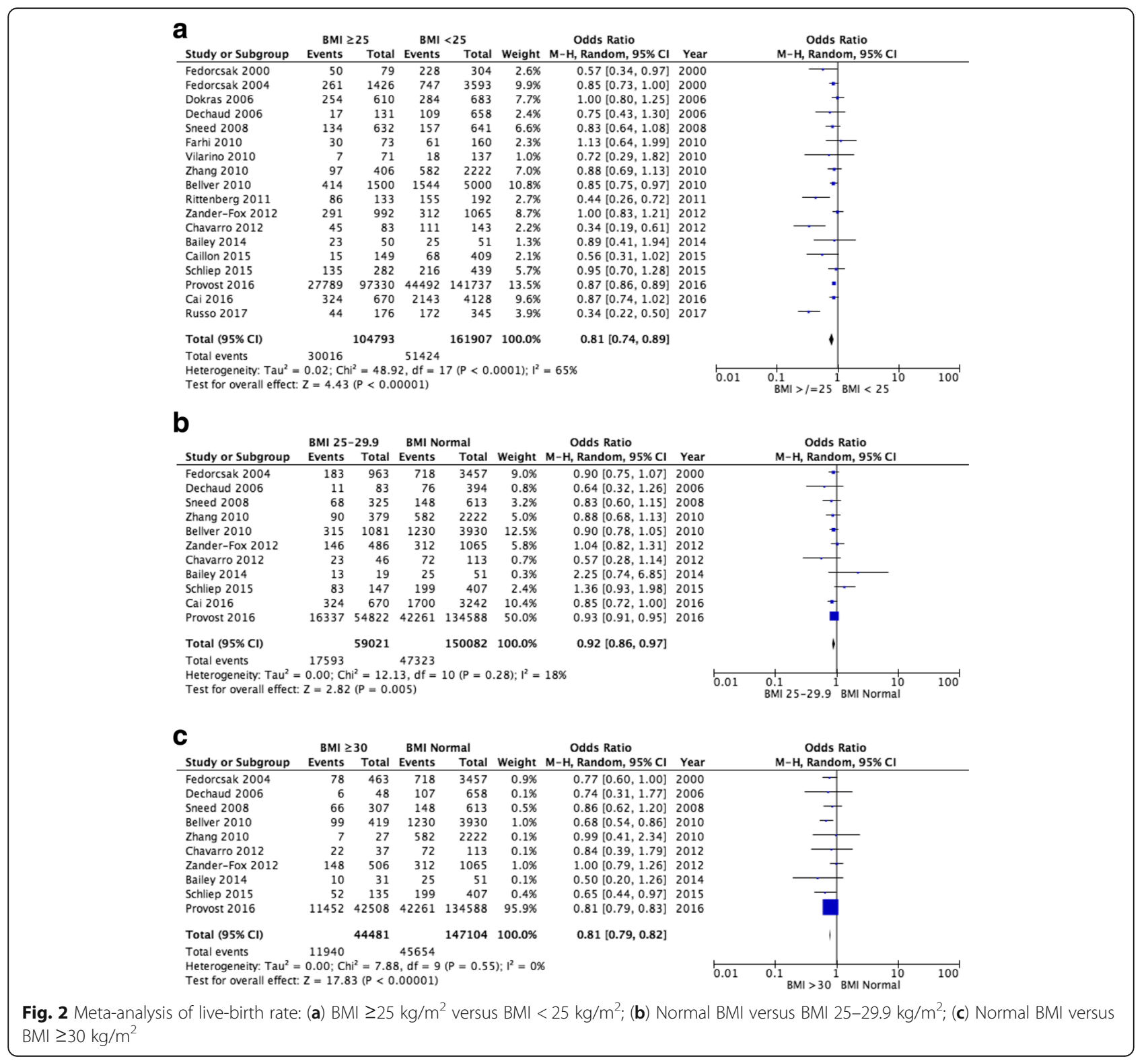




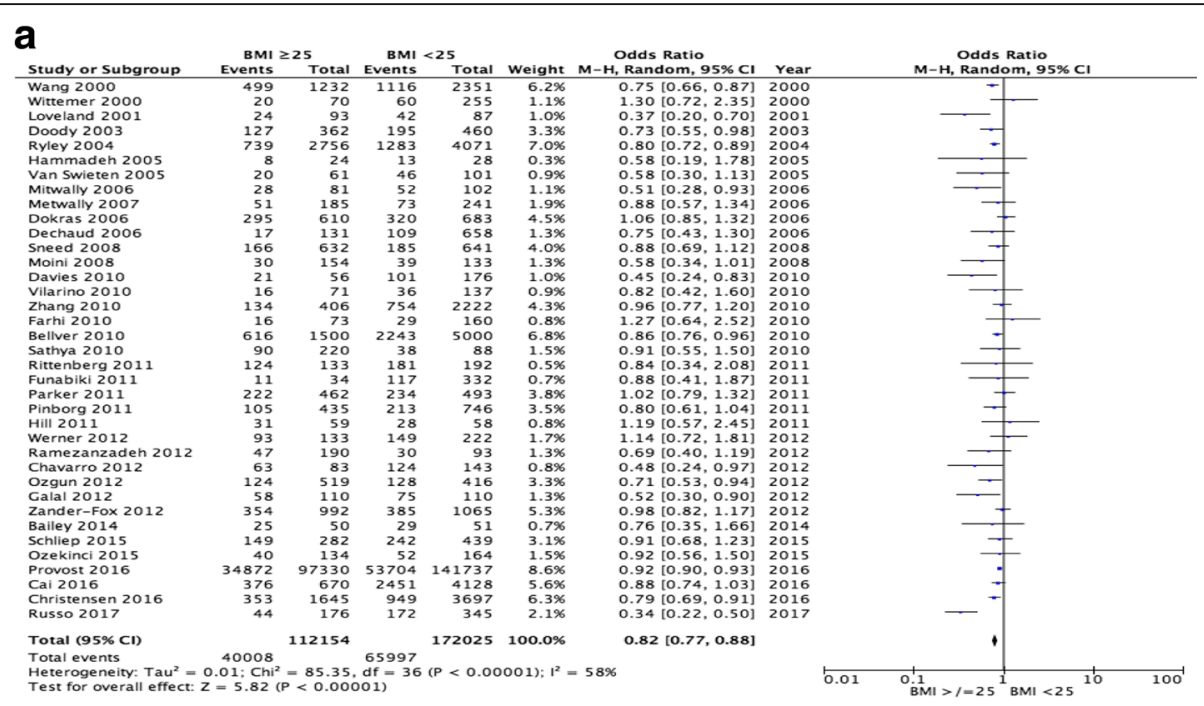

b
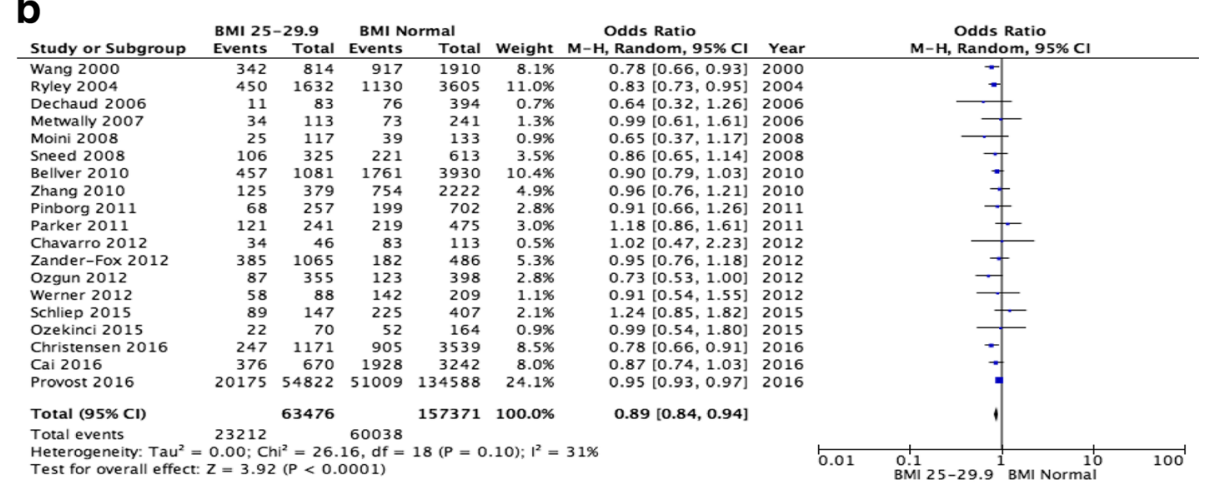

C

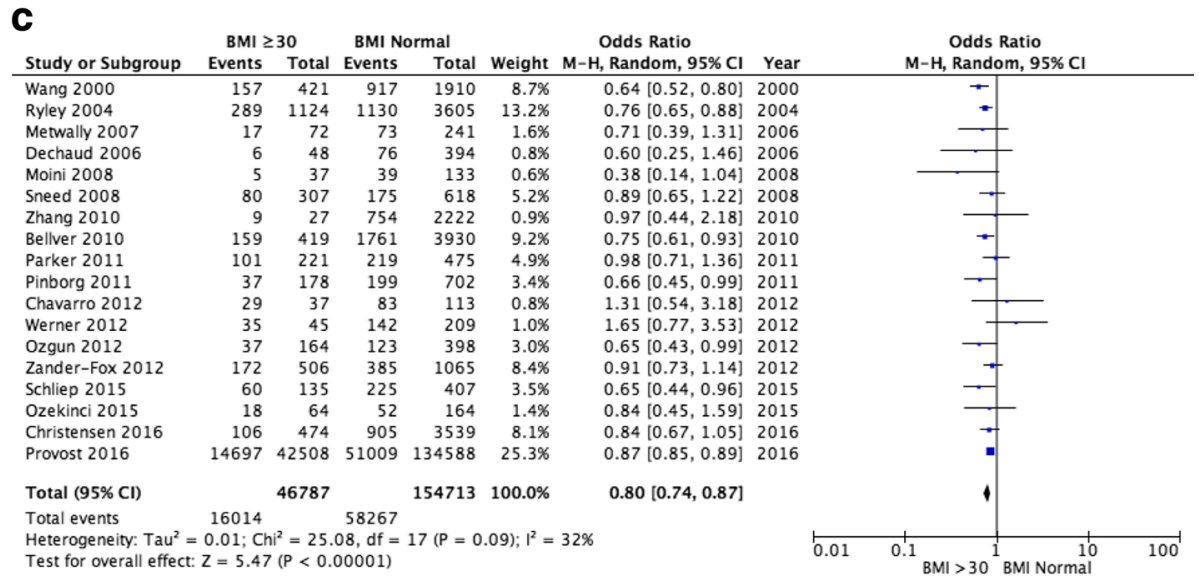

Fig. 3 Meta-analysis of clinical pregnancy rate: (a) BMl $\geq 25 \mathrm{~kg} / \mathrm{m}^{2}$ versus BMl $<25 \mathrm{~kg} / \mathrm{m}^{2}$; (b) Normal BMl versus BMI 25-29.9 kg/m²; (c) Normal BMl versus $B M l \geq 30 \mathrm{~kg} / \mathrm{m}^{2}$

Women with a BMI $25-29.9 \mathrm{~kg} / \mathrm{m}^{2}$ were also more likely to have a miscarriage when compared to women with a normal BMI (18 studies pooled, OR 1.15 95\% CI 1.05-1.26, $p=0.002$; Fig. 4b). There was no significant clinical heterogeneity $\left(\mathrm{I}^{2}=16 \%\right)$ in this group.
The risk of miscarriage is further increased in women with a $B M I \geq 30 \mathrm{~kg} / \mathrm{m}^{2}$ when compared to women who fall into a normal BMI category (17 studies pooled, OR 1.52, 95\% CI 1.28-1.81, $p<0.00001$; Fig. 4c). No significant heterogeneity $\left(\mathrm{I}^{2}=46 \%\right)$ was demonstrated between the studies. 


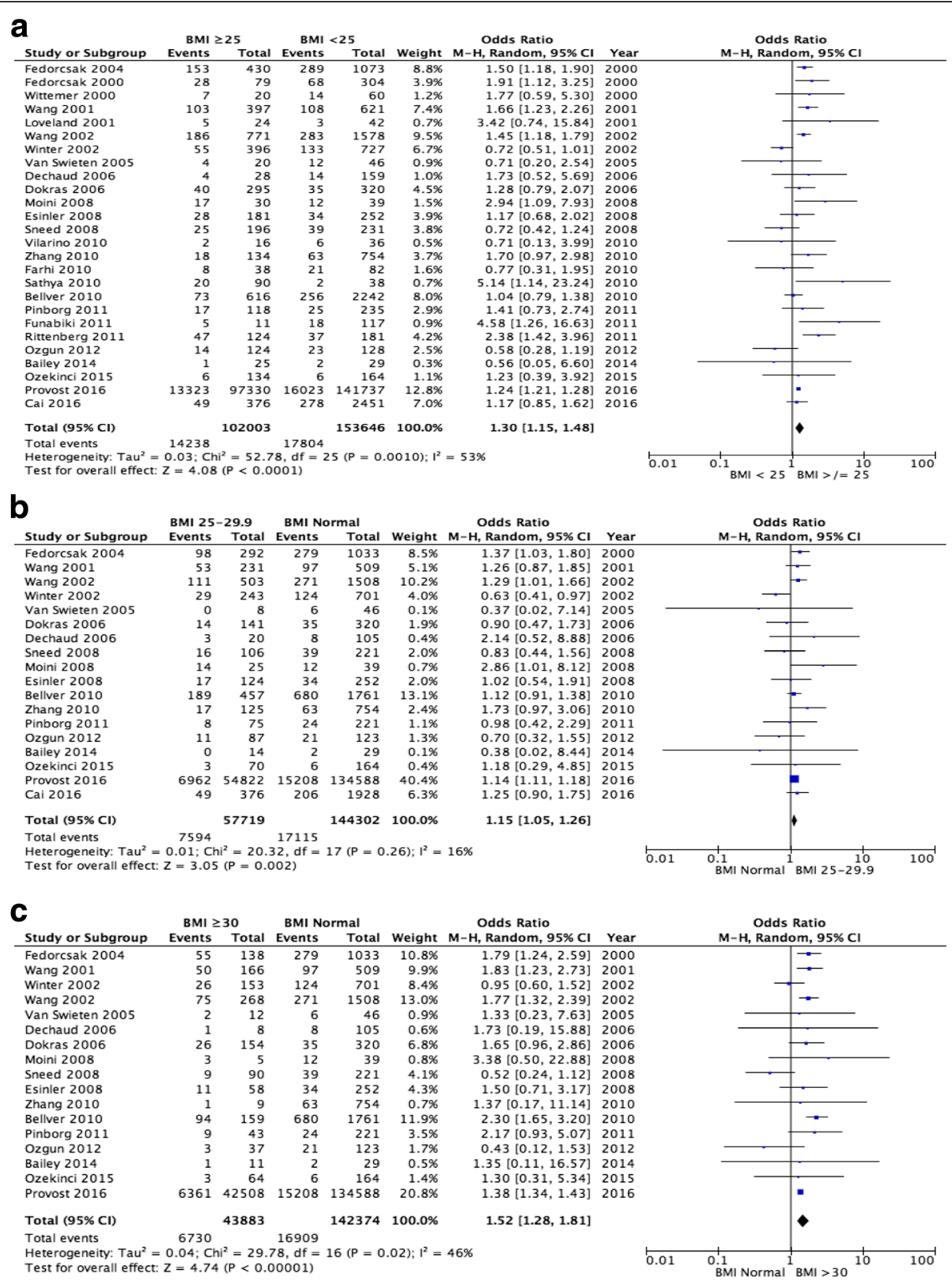

Fig. 4 Meta-analysis of miscarriage rate: (a) BMI $\geq 25 \mathrm{~kg} / \mathrm{m}^{2}$ versus BMl $<25 \mathrm{~kg} / \mathrm{m}^{2}$; (b) Normal BMl versus BMl $25-29.9 \mathrm{~kg} / \mathrm{m}^{2}$; (c) Normal BMl versus $\mathrm{BMI} \geq 30 \mathrm{~kg} / \mathrm{m}^{2}$

\section{Dosage of gonadotrophin stimulation}

Women with a BMI $\geq 25 \mathrm{~kg} / \mathrm{m}^{2}$ required significantly larger total gonadotrophin dosages than women with a BMI $<25 \mathrm{~kg} / \mathrm{m}^{2}$ (15 studies pooled, weighted mean difference [WMD] 196.03iu, 95\% CI 131.91-260.16, $p<0.00001$; Fig. 5a). However, significant heterogeneity $\left(\mathrm{I}^{2}=75 \%\right.$, $p<0.00001)$ was present between the studies.

Women with a BMI $25-29.9 \mathrm{~kg} / \mathrm{m}^{2}$ were demonstrated to require significantly higher total gonadotrophin dosages than women with a normal BMI (12 studies pooled, WMD 83.67iu, 95\% CI 24.54-142.80, $p=0.006$; Fig. 5b). However, significant heterogeneity $\left(\mathrm{I}^{2}=80 \%, p<0.00001\right)$ existed between the studies.

Furthermore, increased total dosages of gonadotrophin was documented for women with a BMI $\geq 30 \mathrm{~kg} / \mathrm{m}^{2}$ when compared to women whose BMI fell into the normal category (13 studies pooled, WMD 363.58iu, 95\% CI 252.99-474.17, p < 0.00001; Fig. 5c). However, significant heterogeneity $\left(\mathrm{I}^{2}=81 \%, p<0.00001\right)$ was present between the studies. 

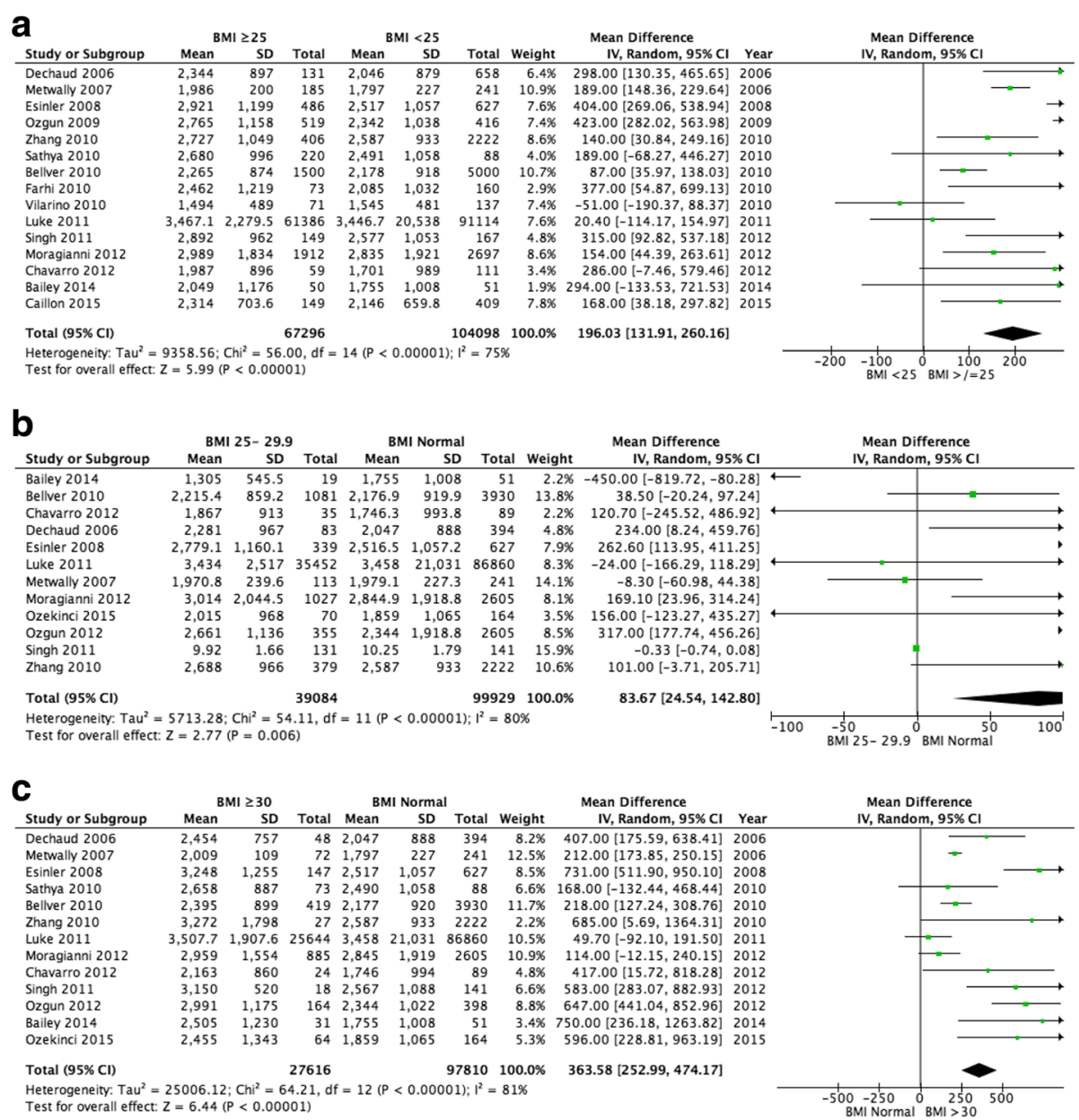

Fig. 5 Meta-analysis of total gonadotrophin dose: (a) BMl $\geq 25 \mathrm{~kg} / \mathrm{m}^{2}$ versus BMl $<25 \mathrm{~kg} / \mathrm{m}^{2}$; (b) Normal BMl versus BMI 25-29.9 $\mathrm{kg} / \mathrm{m}^{2}$; (c) Normal BMI versus BMI $\geq 30 \mathrm{~kg} / \mathrm{m}^{2}$

\section{Duration of gonadotrophin stimulation}

No significant difference in duration of stimulation therapy was documented between women with a BMI $<25 \mathrm{~kg} / \mathrm{m}^{2}$ or $\geq 25 \mathrm{~kg} / \mathrm{m}^{2}$ (13 studies pooled, WMD 0.10, 95\% CI -0.10$0.31, p=0.32$; Fig. $6 \mathrm{a})$, however significant heterogeneity $\left(\mathrm{I}^{2}\right.$ $=95 \%, p<0.00001)$ existed between the included studies.

Furthermore, no significant difference was seen for duration of gonadotrophin stimulation between women with a BMI $25-29.9 \mathrm{~kg} / \mathrm{m}^{2}$ versus a normal BMI ( 8 pooled studies, WMD 0.02, 95\% CI -0.10-0.13, $p=0.79, \mathrm{I}^{2}=48 \%$; Fig. $\left.6 \mathrm{~b}\right)$ or for women with a BMI $\geq 30 \mathrm{~kg} / \mathrm{m}^{2}$ versus a normal BMI (12 pooled studies, WMD 0.12 95\% CI -0.24-0.47, $p=0.52$; Fig. 6c), however significant heterogeneity $\left(\mathrm{I}^{2}=96 \%, p<0.00001\right)$ was noted between the studies for the latter comparison.

\section{Discussion}

Public funding for fertility services within the United Kingdom is limited, and therefore, strict guidance exists regarding who can be offered treatment under the National Health Service (NHS). Body mass index (BMI) is a universal criteria adopted by both the public and private sector. This study addresses an important aspect of the impact of a raised BMI on fertility treatment outcomes.

We standardise the analysis of the data by only including studies incorporating the WHO BMI criteria; the current reference point for clinicians and clinical commissioning groups in ascertaining which group of patients should receive treatment. This study is an update of the previous systematic review performed in 2010 , with the inclusion of a larger number of cycles from central databases such as the Society for Assisted Reproductive Technology (SART).

This systematic review and meta-analysis has clearly highlighted the negative impact of a raised BMI on the outcomes following ART treatment, with documented lower success rates and higher rates of miscarriages as well as higher total dosage of gonadotrophin usage with 


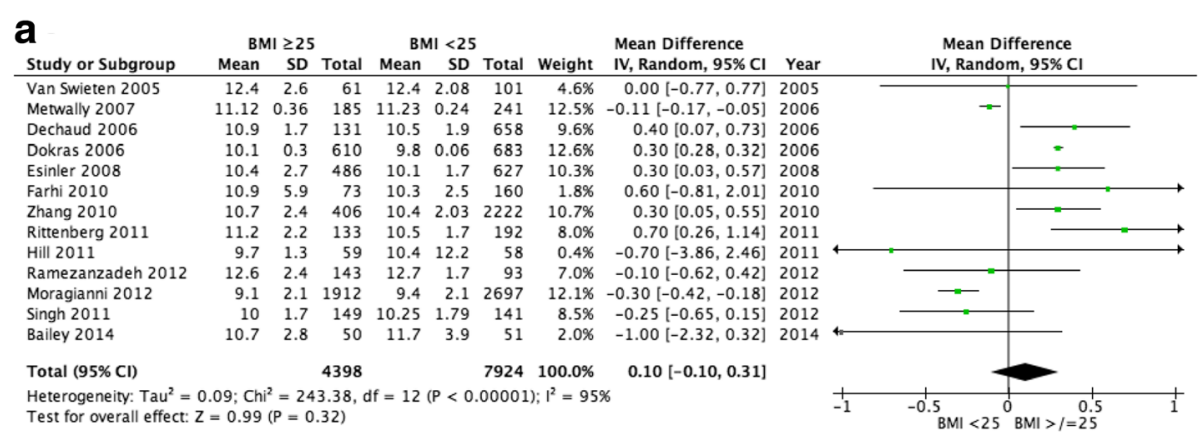

\section{b}
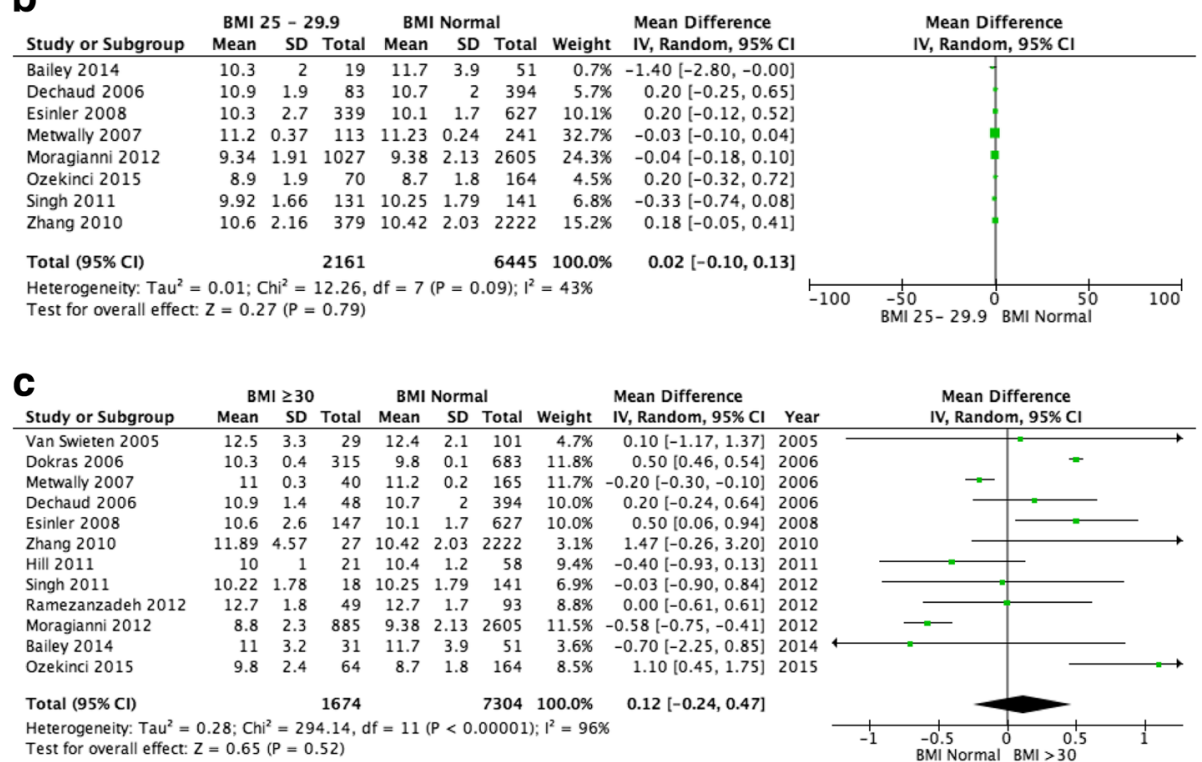

Fig. 6 Meta-analysis of duration of gonadotrophin stimulation: (a) BMl $\geq 25 \mathrm{~kg} / \mathrm{m}^{2}$ versus $\mathrm{BMl}<25 \mathrm{~kg} / \mathrm{m}^{2}$; (b) Normal BMl versus BMI $25-29.9 \mathrm{~kg} / \mathrm{m}^{2}$; (c) Normal BMI versus BMI $\geq 30 \mathrm{~kg} / \mathrm{m}^{2}$

no effect on the duration of stimulation. The latter may have been balanced by higher dosages of treatment which can also have a cost implication. However, as most studies have included a BMI category of $<25 \mathrm{~kg} /$ $\mathrm{m}^{2}$, which would also include underweight women with a BMI $<18 \mathrm{~kg} / \mathrm{m}^{2}$, the detrimental effects of which have been addressed in a number of previous studies, a risk of bias cannot be confidently excluded. This has been addressed through the inclusion of studies allowing for a sub-group analysis of women with a normal BMI with overweight and obese women.

The presented data is able to demonstrate statistical significance with low clinical heterogeneity for a number of factors reflective of success through ART treatment. Despite this, caution is advised for interpretation of the presented information as only a few of the included studies controlled for confounding factors such as age, smoking and duration of infertility. In order to reduce further clinical heterogeneity, studies not incorporating the WHO classification for BMI and paternal BMI were excluded.
The included studies were considered relevant if they conformed to the WHO classification of BMI, despite this, a considerable amount of methodological and clinical heterogeneity existed. The level of statistical heterogeneity for the primary outcome measure live birth rate and secondary outcome measures clinical pregnancy rate and miscarriage rate were limited. However, despite a significant increase in total gonadotrophin dosage requirements with increasing BMI categories, the studies demonstrated significant statistical heterogeneity, limiting their value.

The presented data can act as an aid in the counselling of subjects secondary to a clear impact on ART outcomes being demonstrated across all BMI categories. The evidence supports the government's stringent allocation of funding when resources are significantly limited.

A raised BMI impacts reproductive health at the pre and post embryological stage of development, affecting oocyte quality and the endometrial environment [2].

A recent meta-analysis and systematic review by Best et al., [5] has demonstrated that weight loss can improve 
pregnancy rate and ovulatory status with a trend favouring spontaneous conception. However, these effects have not been seen through ART. Of note, miscarriage rates were unaltered with a change in weight.

Besides the reproductive health effects of a raised BMI, clinicians should also be aware of the increased rate of pregnancy complications such as pregnancy induced hypertension, pre-eclampsia and gestational diabetes in women with a raised BMI. Women are also at an increased risk of an emergency caesarean section with increasing BMI [6, 7].

A holistic approach should be used when counselling patients seeking ART treatments using an open discussion method to inform patients of the effects of raised BMI on ART and obstetric care. This will allow couples to make an informed decision and to take ownership of their well-being.

\section{Conclusion}

This systematic review and meta-analysis further emphasises the negative impact of a raised BMI on ART outcomes. However, the underlying pathophysiology is beyond the scope of this systematic review and will need to be evaluated in future studies. The quality of this systematic review would be further improved if future study designs included the WHO classification of BMI and controlled for confounding variables.

\section{Abbreviations}

ART: Assisted reproductive technology; BMI: Body Mass Index; FSH: Follicle stimulation hormone; GIFT: Gamete intra-Fallopian transfer; HCG: Human chorionic gonadotrophin; ICSI: Intracytoplasmic sperm injection; IVF: In vitro fertilization; MeSH: Medical subject headings; NHS: National Health Service; OHSS: Ovarian hyperstimulation syndrome; SART: Society for Assisted Reproductive Technology

\section{Acknowledgements}

Not applicable.

Funding

Not applicable.

\section{Availability of data and materials}

All data generated or analysed during this study are included in this published article.

\section{Authors' contributions}

PRS and MM analyzed and interpreted the data. LNL developed the initial idea. EM and LNL oversaw the progress of the manuscript. All authors read and approved the final manuscript.

\section{Ethics approval and consent to participate}

Not applicable.

\section{Consent for publication}

Not applicable.

\section{Competing interests}

The authors declare that they have no competing interests.

\section{Publisher's Note}

Springer Nature remains neutral with regard to jurisdictional claims in published maps and institutional affiliations.

\section{Author details}

'Oxford University Hospitals NHS Foundation Trust, John Radcliffe Hospital, Headley Way, Headington, Oxford OX3 9DU, UK. ${ }^{2}$ Nuffield Department of Women's and Reproductive Health, University of Oxford, Level 3, Women's Centre, John Radcliffe Hospital, Oxford OX3 9DU, UK.

Received: 1 May 2017 Accepted: 18 February 2018

Published online: 27 February 2018

\section{References}

1. WHO. Obesity and overweight. Geneva: World Health Organization. Fact Sheet 311. Available from http://www.who.int/mediacentre/factsheets/ fs311/en/.

2. Rittenberg V, Seshadri S, Sunkara SK, Sobaleva S, Oteng-Ntim E, El-Toukhy T. Effect of body mass index on IVF treatment outcome: an updated systematic review and meta-analysis. Reprod BioMed Online. 2011;23(4): 421-39.

3. Higgins JPT, Thompson SG. Quantifying heterogeneity in a meta-analysis. Stat Med. 2002;21(11):1539-58.

4. Mantel N, Haenszel W. Statistical aspects of the analysis of data from retrospective studies of disease. J Natl Cancer Inst. 1959;22:719-48.

5. Best D, Avenell A, Bhattacharya S. How effective are weight-loss interventions for improving fertility in women and men who are overweight or obese? A systematic review and meta-analysis of the evidence. Hum Reprod Update. 2017;23(6):681-705.

6. Aly H, Hammad T, Nada A, Mohamed M, Bathgate S, El-Mohandes A. Maternal obesity, associated complications and risk of prematurity. J Perinatol. 2010;30(7):447-51.

7. Bhattacharya S, Campbell DM, Liston WA, Bhattacharya S. Effect of body mass index on pregnancy outcomes in nulliparous women delivering singleton babies. BMC Public Health. 2007;7:168.

8. Fedorcsak $P$, et al. Obesity is a risk factor for early pregnancy loss after IVF or ICSI. Acta Obstet Gynecol Scand. 2000;79(1):43-8.

9. Wittemer $C$, et al. Does body mass index of infertile women have an impact on IVF procedure and outcome? J Assist Reprod Genet. 2000;17(10):547-52.

10. Wang JX, Davies M, Norman RJ. Body mass and probability of pregnancy during assisted reproduction treatment: retrospective study. Br Med J. 2000; 321(7272):1320-1.

11. Loveland $J B$, et al. Increased body mass index has a deleterious effect on in vitro fertilization outcome. J Assist Reprod Genet. 2001;18(7):382-6.

12. Wang JX, Davies MJ, Norman RJ. Polycystic ovarian syndrome and the risk of spontaneous abortion following assisted reproductive technology treatment. Hum Reprod. 2001;16(12):2606-9.

13. Wang $X$, Davies J, Norman J. Obesity increases the risk of spontaneous abortion during infertility treatment. Obes Res. 2002;10(6):551-4.

14. Winter E, Wang J, Davies MJ, Norman RJ. Early pregnancy loss following assisted reproductive technology treatment. Hum Reprod. 2002;12:3220-3.

15. Doody KM, Langley MT, Marek DE, Nackley AC, Doody KJ. Morbid obesity adversely impacts outcomes with IVF. Fertility Sterility. 2003;80:S160.

16. Fedorcsák P, Dale PO, Storeng R, Ertzeid G, Bjercke S, Oldereid N, Omland AK, Abyholm T, Tanbo T. Impact of overweight and underweight on assisted reproduction treatment. Hum Reprod. 2004;19(11):2523-8.

17. Ryley DA, Bayer SR, Eaton J, Zimon A, Klipstein S, Reindollar. Influence of body mass index (BMI) on the outcome of 6,827 IVF cycles. Fertil Steril. 2004;82(Supplement 2):S38-9.

18. Van ECAM, et al. Obesity and clomiphene challenge test as predictors of outcome of in vitro fertilization and intracytoplasmic sperm injection. Gynecol Obstet Investig. 2005;59(4):220-4.

19. Hammadeh ME, Sykoutris A, Amer AS, Schmidt. Relationship between body mass index (BMI) and plasma lipid concentration and their effect on IVF/ICSI outcome. Fertil Steril. 2005:84(Supplement 1):S422.

20. Dechaud $\mathrm{H}$, et al. Obesity does not adversely affect results in patients who are undergoing in vitro fertilization and embryo transfer. European Journal of Obstetrics Gynecology and Reproductive Biology. 2006;127(1):88-93.

21. Dokras A, et al. Obstetric outcomes after in vitro fertilization in obese and morbidly obese women. Obstet Gynecol. 2006;108(1):61-9. 
22. Mitwally MF, Leduc MM, Ogunleye O, Albuarki H, Diamond MP, Abuzeid M. The effect of body mass index (BMI) on the outcome of IVF and embryo transfer in women of different ethnic backgrounds. Fertility Sterility. 2006;86:S68-9.

23. Metwally M, Cutting R, Tipton A, Skull J, Ledger WL, Li TC. Effect of increased body mass index on oocyte and embryo quality in IVF patients. Reprod BioMed Online. 2007;15:532-8.

24. Esinler I, Bozdag G, Yarali H. Impact of isolated obesity on ICSI outcome. Reprod BioMed Online. 2008;17(4):583-7.

25. Martinuzzi $\mathrm{K}$, et al. Elevated body mass index (BMI) does not adversely affect in vitro fertilization outcome in young women. J Assist Reprod Genet. 2008; 25(5):169-75.

26. Moini A, et al. The effect of body mass index on the outcome of IVF/ICSI cycles in non polycystic ovary syndrome women. International Journal of Fertility and Sterility. 2008;2(2):82-5.

27. Sneed ML, et al. Body mass index: impact on IVF success appears agerelated. Hum Reprod. 2008;23(8):1835-9.

28. Ozgun MT, et al. The influence of body mass index on FSH dose and pregnancy rate in women undergoing ICSI-embryo transfer. Journal of the Turkish German Gynecology Association. 2009;10(1):1-5.

29. Sathya A, et al. Effect of body mass index on in vitro fertilization outcomes in women. Journal of Human Reproductive Sciences. 2010;3(3):135-8.

30. Zhang $D$, et al. Overweight and obesity negatively affect the outcomes of ovarian stimulation and in vitro fertilisation: a cohort study of 2628 Chinese women. Gynecol Endocrinol. 2010;26(5):325-32.

31. Bellver J, et al. Female obesity impairs in vitro fertilization outcome without affecting embryo quality. Fertil Steril. 2010;93(2):447-54.

32. Vilarino Lima F, et al. Body mass index and fertility: is there a correlation with human reproduction outcomes? Gynecol Endocrinol. 2011;27(4):232-6.

33. Farhi J, et al. High-quality embryos retain their implantation capability in overweight women. Reprod BioMed Online. 2010;21(5):706-11.

34. Davies $D$, et al. Are the negative effects of being overweight on reproductive potential evident in FET cycles? Reprod BioMed Online. 2010; 20(Supplement S2):S58.

35. Funabiki $\mathrm{M}$, et al. The influence of body mass index (BMI) on pregnancy outcomes among Japanese infertile women. Fertil Steril. 2011;96(3 Supplement 1):S115.

36. Hill MJ, Hong S, Frattarelli JL. Body mass index impacts in vitro fertilization stimulation. ISRN Obstetrics and Gynecology. 2011:2010:1-5.

37. Pinborg A, et al. Influence of female bodyweight on IVF outcome: a longitudinal multicentre cohort study of 487 infertile couples. Reprod BioMed Online. 2011;23(4):490-9.

38. Parker $\mathrm{K}$, et al. Does body mass index (BMI) affect IVF outcomes? Fertil Steril. 2011;96(3 Supplement 1):S124.

39. Rittenberg $\mathrm{V}$, Sobaleva $\mathrm{S}$, et al. Influence of BMI on risk of miscarriage after single blastocyst transfer. Hum Reprod. 2011;26(10):2642-50.

40. Singh $\mathrm{N}$, et al. Correlation of body mass index with outcome of in vitro fertilization in a developing country. Arch Gynecol Obstet. 2012;285(1):259-63.

41. Luke B, et al. Female obesity adversely affects assisted reproductive technology (ART) pregnancy and live birth rates. Hum Reprod. 2011;26(1): 245-52.

42. Chavarro JE, et al. Body mass index and short-term weight change in relation to treatment outcomes in women undergoing assisted reproduction. Fertil Steril. 2012;98(1):109-16.

43. Galal AF, Elhelaly DN. Higher body mass index significantly affects intracytoplasmic sperm injection success rate. Fertil Steril. 2012:97(3 Supplement 1):S9-S10.

44. Werner $\mathrm{M}$, et al. Body mass index (BMI) does not impact endometrial receptivity in fresh IVF cycles: evaluation of implantation rates (IR) and ongoing pregnancy rates (PR) following the transfer of euploid blastocysts. Fertil Steril. 2012;98(3 Supplement 1):S286-7.

45. Zander-Fox DL, et al. Does obesity really matter? The impact of BMI on embryo quality and pregnancy outcomes after IVF in women aged $<38$ years. Aust N Z J Obstet Gynaecol. 2012;52(3):270-6.

46. Ozgun MT, et al. Effect of body mass index on the risk of miscarriage after ICSI. Int J Gynecol Obstet. 2012;119:S535.

47. Ramezanzadeh F, et al. Impact of body mass index versus physical activity and calorie intake on assisted reproduction outcomes. European Journal of Obstetrics Gynecology and Reproductive Biology. 2012;163(1):52-6.

48. Moragianni VA, Jones SML, Ryley DA. The effect of body mass index on the outcomes of first assisted reproductive technology cycles. Fertil Steril. 2012; 98(1):102-8.
49. Bailey AP, et al. Effect of body mass index on in vitro fertilization outcomes in women with polycystic ovary syndrome. Am J Obstet Gynecol. 2014; 211(2):163.e1-6.

50. Schliep KC, et al. Effect of male and female body mass index on pregnancy and live birth success after in vitro fertilization. Fertil Steril. 2015;103(2):388-95.

51. Cai J, Liu L, Zhang J, Qiu H, Jiang X, Li P, Sha A, Ren J. Low body mass index compromises live birth rate in fresh transfer in vitro fertilization cycles: a retrospective study in a Chinese population. Fertility Sterility. 2017;107(2): 422-9.

52. Ozekinci M, Seven A, Olgan S, Sakinci M, Keskin U, Akar ME, Ceyhan ST, Ergun A. Does obesity have detrimental effects on IVF treatment outcomes? BMC Womens Health. 2015;15:61. https://doi.org/10.1186/s12905-015-0223-0.

53. Caillon H, Fréour T, Bach-Ngohou K, Colombel A, Denis MG, Barrière P, Masson D. Effects of female increased body mass index on in vitro fertilization cycles outcome. Obesity Research \& Clinical Practice. 2015;9: 382-8.

54. Provost MP, Acharya KS, Acharya CR, Yeh JS, Steward JG, Eaton JL, Goldfarb JM, Muasher SJ. Pregnancy outcomes decline with increasing body mass index: analysis of 239,127 fresh autologous in vitro fertilization cycles from the 2008-2010 Society for Assisted Reproductive Technology registry. Fertil Steril. 2016;105(3):663-9.

55. Russo M, Ates S, Shaulov T, Dahan MH. Morbid obesity and pregnancy outcomes after single blastocyst transfer: a retrospective, north American study. J Assist Reprod Genet. 2017; https://doi.org/10.1007/s10815-017-0883-9.

56. Christensen MW, Ingerslev HJ, Degn B, Kesmodel US. Effect of female body mass index on oocyte quantity in fertility treatments (IVF): treatment cycle number is a possible effect modifier. A Register-Based Cohort Study PLoS ONE. 2016;11(9):e0163393. https://doi.org/10.1371/journal.

\section{Submit your next manuscript to BioMed Central and we will help you at every step:}

- We accept pre-submission inquiries

- Our selector tool helps you to find the most relevant journal

- We provide round the clock customer support

- Convenient online submission

- Thorough peer review

- Inclusion in PubMed and all major indexing services

- Maximum visibility for your research

Submit your manuscript at www.biomedcentral.com/submit
Biomed Central 